\title{
Suitable Ultrasonographic Approach to Assessing Vocal Cord Movement: Reply
}

\author{
Takahiro Fukuhara ${ }^{1}$
}

Published online: 13 November 2017

(C) Société Internationale de Chirurgie 2017

In this study, there was a significant difference in the visualization rate between the conventional transverse procedure and the lateral vertical procedure (70.2 vs. 98.4\%) [1]. Therefore, we did not bother to mention the sensitivity, specificity, diagnostic accuracy, positive predictive value (PPV), or negative predictive value (NPV), largely to prevent comparative advantages and disadvantages of these procedures from being misconstrued. As an alternative, we described the detailed results in Table 2 [1].

When only the visible cases were analyzed, the sensitivity, specificity, diagnostic accuracy, PPV, and NPV were $95.2,100,99.2,100$, and $99.1 \%$, respectively, for the conventional middle transverse procedure, and 66.7, 100, $93.5,100$, and $92.5 \%$, respectively, for the novel lateral vertical procedure [1].

Ultrasonographic criteria were described in the section entitled 'Measurement procedure' (paragraph 3, lines 7-16) [1]. We judged only positive or negative findings by ultrasonography and determined vocal cord paresis or paralysis by laryngoscopy. Table 2 is described in the text (Results; paragraph 3, lines 1-3) [1].

We also believe that laryngoscopy is the preferred method for detailed examination of vocal cord movement, as stated in the text, whereas ultrasonography may be useful for screening for vocal cord paresis or paralysis [1]. Furthermore, we believe that a suitable approach to ultrasonographic assessment for vocal cord movement would be to first evaluate vocal cord movement by the conventional method and then evaluate it by our lateral vertical method.

\section{Reference}

1. Fukuhara T, Donishi R, Matsuda E, Koyama S, Fujiwara K, Takeuchi H (2017) A novel lateral approach to the assessment of vocal cord movement by ultrasonography. World J Surg. https:// doi.org/10.1007/s00268-017-4151-z
Takahiro Fukuhara

tfukuhara3387@med.tottori-u.ac.jp

1 Department of Otolaryngology-Head and Neck Surgery, Tottori University Faculty of Medicine, 36-1, Nishicho, Yonago 683-8504, Japan 